\title{
Editorial
}

\section{Long axis function in disease}

Although longitudinally directed fibres - situated mainly in the subepicardium and subendocardium regions of the left and right ventricular free walls and the papillary musclescomprise only a small proportion of the total ventricular myocardial mass, they play a major role in the maintenance of normal ejection fraction and in determining atrioventricular interactions. ${ }^{1}$ Not surprisingly, therefore, loss of longitudinal fibre function leads to characteristic disturbances.

\section{Systolic function}

Longitudinal function is always reduced when ventricular cavity size is increased, in addition ejection fraction is reduced and may be absent. ${ }^{23}$ This relation is consistent enough for long axis amplitude, or its equivalent, the amplitude of atrioventricular ring motion, to be used as an index of ejection fraction. ${ }^{4}$ It applies not only to the left ventricle, where it can be shown to relate to prognosis but also to the right, where it provides a simple method of assessing right ventricular function. ${ }^{5}$ When overall long axis amplitude is low, peak shortening and lengthening rates are reduced. In restrictive left ventricular disease, long axis amplitude is low even when cavity size is normal at end diastole, although the effects of this reduction are apparent in a reduced amplitude of wall thickening and thus of shortening fraction. ${ }^{6}$ After mitral valve replacement, long axis amplitude is strikingly reduced; this does not occur with mitral valve repair or mild mitral stenosis, ${ }^{7}$ nor is it a consistent effect of cardiopulmonary bypass done for other reasons, and so is likely to be the result of loss of papillary muscle function. Although shortening fraction is frequently normal in such patients, normal wall thickening is associated with an exaggerated amplitude of epicardial motion, possibly to compensate for loss of the component owing to long axis shortening.

Regional reduction in the extent and velocity of long axis shortening is common after myocardial infarction. Anterior $\mathrm{Q}$ waves correlate with septal involvement, and inferior $\mathrm{Q}$ waves with posterior infarction with or without a right ventricular component. These disturbances correlate closely with the presence of a fixed defect on myocardial perfusion scanning. ${ }^{8}$

\section{Uncoordinated contraction}

A feature of long axis function as distinct from that of the minor axis, as well as cavity volume and pressure, is the frequency with which the timing rather than the amplitude or velocity of motion is abnormal. These disturbances span the conventional periods of systole and diastole, and the rapid repetition rate of $M$ mode echocardiography has proved particularly useful for their study. So that abnormal timing can be appreciated, it is essential that some reference point is recorded early in diastole, reflecting the function of the ventricle as a whole. A simple phonocardiogram demonstrating the second heart sound has proved suitable.

\section{Coronary artery disease}

Asynchronous left ventricular long axis function is common in patients with chronic stable coronary artery disease and is segmental in its distribution. ${ }^{9}$ In affected areas, the onset of contraction is delayed and may be replaced by abnormal lengthening during isovolumic contraction and early ejection. In spite of this, peak shortening velocity is usually normal but contraction continues after A2, so that the onset of lengthening may be delayed until the onset of atrial systole. ${ }^{10}$ Long axis shortening during isovolumic relaxation is often associated with premature minor axis lengthening, so that ventricular cavity shape changes during isovolumic relaxation. The overall effect of this asynchrony is to reduce or even suppress the early diastolic E wave on transmitral Doppler, and to increase the amplitude of the A wave. These changes in filling pattern are traditionally ascribed to "abnormal relaxation". ${ }^{11}$ Although the exact nature of the disturbance invoked is rarely defined, it appears that asynchrony of this kind, which has its origin much earlier in the cardiac cycle, is in fact much the most common cause of depression of peak $\mathrm{E}$ wave velocity below the age related normal value. Classic measurements of diastolic function, such as isovolumic relaxation time or the $t^{1 / 2}$ of pressure fall are unable to distinguish between incoordination and a generalised reduction in the relaxation rate of individual muscle fibres.

This asynchrony may be related directly to coronary artery stenosis. It is aggravated by balloon inflation during coronary angioplasty, ${ }^{12}$ and invariably resolves within 48 hours of a successful procedure. ${ }^{13}$ The presence and location of asynchrony correlates closely with reversible abnormalities on thallium myocardial perfusion scanning. ${ }^{8}$ Asynchrony occurs in the absence of symptoms or ECG changes, and thus provides the most sensitive non-invasive evidence of ischaemia. Its extent is greatly aggravated by dobutamine stress. ${ }^{14}$ It is probably closely related to disturbances previously described by angiography, ${ }^{15}$ and to local asynchrony of wall dynamics demonstrable by transoesophageal echocardiography immediately before vein grafting, which revert within four hours of operation. ${ }^{16}$ This remarkable sensitivity of long axis function to ischaemia may well be the effect of a subendocardial position of a substantial proportion of longitudinally directed fibres.

\section{Activation abnormalities}

Long axis motion is also sensitive to abnormal ventricular activation, possibly again reflecting the subendocardial location of longitudinally directed fibres. Simple right bundle branch block delays the onset of right ventricular long axis shortening by $60 \mathrm{~ms}$ with respect to the ECG. On the left side of the heart, both the start and the end of septal long axis shortening are delayed with an ECG pattern of left bundle branch block (LBBB) (fig 1), so that both isovolumic contraction and isovolumic relaxation become uncoordinated. ${ }^{17}$ These changes vary with activation pattern in intermittent $\mathrm{LBBB}$, demonstrating that they are not the simple consequence of septal fibrosis or other structural abnormality. Septal asynchrony consistently correlates with absence of the septal Q wave on 12 lead 


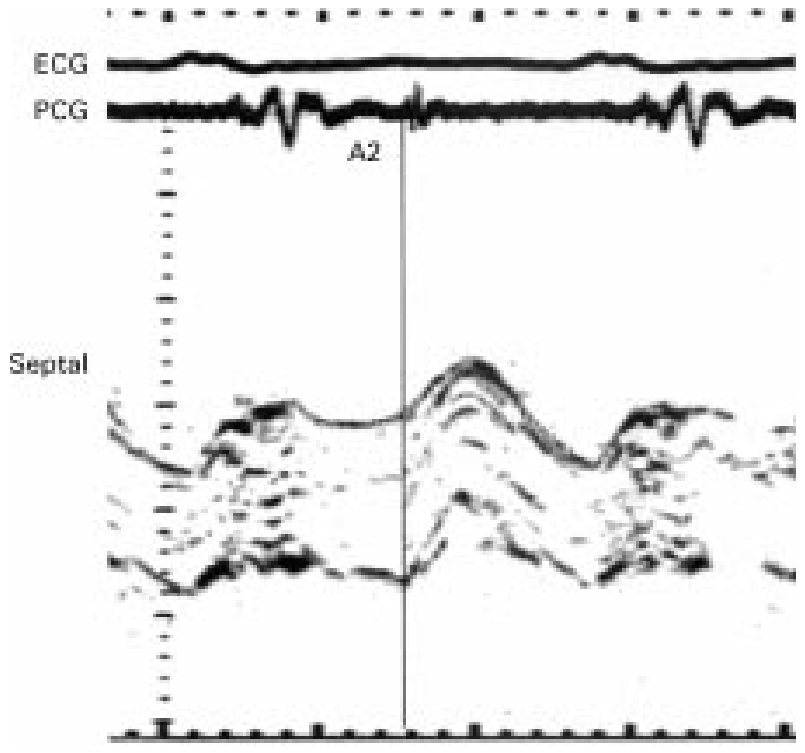

Figure 1 Septal long axis trace in a patient with $L B B B$ showing abnormal lengthening during systole and shortening during early diastole.

ECG, a pattern long known to be directly correlated with septal fibrosis. ${ }^{18}$ Again septal contraction is prolonged during isovolumic relaxation and Doppler $\mathrm{E}$ wave amplitude is reduced, demonstrating interrelations between ventricular activation and what is classically described as "abnormal relaxation". ${ }^{11}$ Ventricular long axis provides a major means of investigating the consequences of abnormalities of activation, particularly in patients with severe ventricular disease. It is likely that this method will be useful in analysing the effects of different modes of ventricular pacing as they are used in the treatment of heart failure.

\section{Left ventricular hypertrophy}

It has long been recognised that left ventricular diastolic function may be abnormal in patients with left ventricular hypertrophy at a time when systolic function is preserved. This has been documented by contrast and nuclear angiography ${ }^{19}$ and by $\mathrm{M}$ mode echocardiography. ${ }^{20}$ All these methods demonstrate that the velocity of early diastolic filling is reduced, with or without superimposed asynchrony. These findings are mirrored in long axis function, when both extent and peak velocity of early diastolic lengthening are reduced and that during atrial systole is increased. ${ }^{21}$ The overall amplitude of motion is, however, normal. In left ventricular hypertrophy, therefore, long axis function corresponds closely to conventional views of diastolic function, and in these circumstances it may be appropriate to calculate the ratio of early to late diastolic lengthening. It is also reasonable to suggest that peak diastolic lengthening rate determined by tissue Doppler may be an index of early diastolic function. ${ }^{22}$ However, these conclusions are limited to cases without asynchrony. In addition, much the most common cause of a reduction of early diastolic lengthening rate is a low overall amplitude of ring motion, which is characteristic of systolic left ventricular disease. Considering peak velocities of long axis motion in isolation, disregarding overall amplitude and timing is thus likely to lead to misleading conclusions.

\section{Atrial function}

Contraction of the pectinate muscles of the atria directly causes atrioventricular ring motion, ${ }^{23}$ thus providing an additional means of studying atrial systolic function. Atrioventricular ring motion appears to be the earliest mechanical consequence of atrial contraction that can be detected non-invasively. Unlike transmitral Doppler, atrioventricular valve motion, or pressure measurements, ring motion reflects local function more precisely. Thus the degree of asynchrony induced by right atrial appendix pacing can be documented and interatrial conduction delay can be measured. The more rapid regression of right atrial function after DC shock for atrial fibrillation with respect to that of the left atrium can also be documented. In addition, the indirect effects of ventricular contraction on atrial function are modified in disease. In particular, when ejection fraction is low, atrioventricular ring motion is reduced so that the systolic increase in atrial capacity falls and with it the systolic component of pulmonary venous flow. ${ }^{24}$

\section{Conclusions}

To the practising cardiologist, the amplitude of long axis or atrioventricular ring motion provides a useful approximation of the ejection fraction of both ventricles, which can rapidly be measured in most patients. Of greater theoretical interest is the insight that studying long axis function provides to the analysis of incoordination and to the investigation of its consequences. In particular, it demonstrates that in most patients so called relaxation abnormalities are in fact the result of incoordination and have their origins much earlier in the cardiac cycle. The frequent involvement of either abnormal activation or coronary artery disease in this incoordination is probably the direct consequence of the subendocardial position of a substantial proportion of longitudinally directed fibres. The close interaction between long axis function and coronary artery disease makes it possible to study the effects of subclinical ischaemia on local and overall ventricular function. In more general terms, observations in disease have emphasised the importance of normal long axis function in maintaining coordinated ventricular contraction. They also begin to indicate the consequences of its loss, as reflected in more conventional measurements of systolic and diastolic function. Long axis function can readily be measured noninvasively, particularly by $\mathrm{M}$ mode or tissue Doppler. We emphasise, however, the importance of determining amplitude, velocity, and timing. Isolated measurements of any of these is likely to prove misleading, but together they appear to provide a substantial addition to currently used methods of assessing ventricular function.

MICHAEL Y HENEIN DEREK G GIBSON

Royal Brompton Hospital,

Sydney Street,

London SW3 6NP, UK

1 Henein MY, Gibson DG. Normal long axis function. Heart 1999;81:111-13. 2 Alam M, Hoglund C, Thorstrand C, et al. Atrioventricular plane displacement in severe congestive heart failure following dilated cardiomyopathy or myocardial infarction. F Intern Med 1990;228:569-75.

3 Brecker SID Xiao HB, Mbaissouroum M et al. Effects of intravenous milrinone on left ventricular function in ischemic and idiopathic dilated rinone on left ventricular function in ische

4 Pai RG, Bodenheimer MM, Pai SM, et al. Usefulness of systolic excursion of the mitral annulus as an index of left ventricular systolic function. $A m \mathcal{F}$ Cardiol 1990;67:222-4.

5 Kaul S, Tei C, Hopkins JM, et al. Assessment of right ventricular function using two-dimensional echocardiography. Am Heart f 1984;107:526-31.

6 Henein MY, Gibson DG. Abnormal subendocardial function in restrictive left ventricular disease. Br Heart $\mathcal{F}$ 1994;72:237-42.

Jones CJH, Raposo L, Gibson DG. Functional importance of the long axis dynamics of the human left ventricle. Br Heart $\mathcal{F} 1990 ; 63: 215-20$.

8 Henein MY, Anagnostopoulos C, Das SK, et al. Left ventricular long axis disturbances as predictors for thallium perfusion defects in patients with known peripheral vascular disease. Heart 1998;79:295-300.

9 Henein MY. Ventricular long axis function in coronary artery disease. Imperial College, National Heart \& Lung Institute, London University: PhD thesis, 1996

10 Henein MY, Gibson DG. Suppression of left ventricular early diastolic filling by long axis asynchrony. Br Heart f 1995;73:151-7.

11 Nagueh SF, Middleton KJ, Kopelen HA, et al. Doppler tissue imaging: a non invasive technique for evaluation of left ventricular relaxation and invasive technique for evaluation of left ventricular relaxation

12 Henein MY, O'Sullivan C, Davies S, et al. Comparison of the effects of acute coronary occlusion and prior ischaemic injury on left ventricular wall motion in humans. Heart 1997;77:338-45. 
13 Henein MY, Priestley K, Davarashvili T, et al. Early changes in left ventricular subendocardial function after successful coronary angioplasty. Br Heart 7 1993;69:501-6.

14 O'Sullivan CA, Henein MY, Sutton R, et al. Abnormal ventricular activation and repolarization during dobutamine stress echocardiography in coronary artery disease. Heart 1998;79:468-73.

15 Gibson DG, Prewitt TA, Brown DJ. Analysis of left ventricular wall movement during isovolumic relaxation and its relation to coronary artery disease. Br Heart f 1976;38:1010

16 Koh TW, Pepper JR, Gibson DG. Early changes in left ventricular anterior wall dynamics and coordination after coronary artery surgery. Heart 1997; 78:291-7.

17 Xiao HB, Roy C, Gibson DG. Nature of ventricular activation in patients with dilated cardiomyopathy. Br Heart f 1994;72:167-74

18 Xiao HB, Gibson DG. Absent septal $\mathrm{Q}$ wave: a marker of the effects of abnormal activation pattern on left ventricular diastolic function. $\mathrm{Br}$ Heart f 1994;72:45-51.
19 Spirito P, Maron B, Bonow RO. Noninvasive assessment of left ventricular diastolic function: comparative analysis of Doppler echocardiographic and radionuclide angiographic techniques. $\mathcal{F} \mathrm{Am}$ Coll Cardiol 1986;7:518-26.

20 Shapiro LM, Gibson DG. Patterns of diastolic dysfunction in left ventricular hypertrophy. Br Heart f 1988;59:438-45.

21 Henein MY, Xiao HB, Brecker SJD, et al. Bernheim "a" wave: obstructed right ventricular inflow or atrial cross talk? Br Heart f 1993;69:409-13.

22 Rodrigues L, Garcia M, Ares M, et al. Assessment of mitral annular dynamics during diastole by Doppler tissue imaging: comparison with mitral Doppler inflow in subjects without heart disease and in patients with left ventricular hypertrophy. Am Heart f 1996;131:982-7.

23 Wang K, Ho SY, Gibson DG, et al. Architecture of atrial musculature in humans. Br Heart $\mathcal{f}$ 1995;73:559-65.

24 Keren G, Sonnenblick EH, LeJemtel TH. Mitral anulus motion. Relation to pulmonary venous and transmitral flows in normal subjects and in patients with dilated cardiomyopathy. Circulation 1988;78:621-9.

\section{STAMPS IN CARDIOLOGY}

\section{Echocardiography}

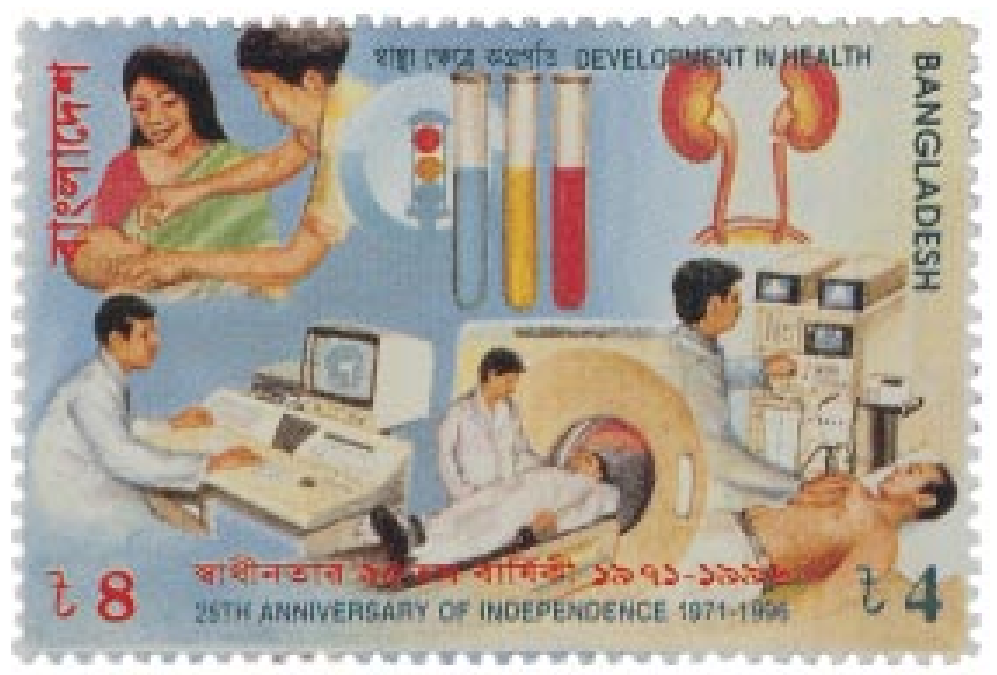

The only stamp that features echocardiography was issued by Bangladesh in 1996 to commemorate the 25th anniversary of independence. The stamp is inscribed "Development in health" and shows immunisation and computed tomography as well as echocardiography.

M K DAVIES A HOLLMAN 